\title{
Smoggy days are here again
}

\author{
Norman L Jones MD, Editor-in-Chief, Canadian Respiratory Journal
}

$\mathrm{M}$ any readers of the Canadian Respiratory Journal know that Hamilton, Ontario, is built on two levels the main city core and industry on the shore of Lake Ontario ('by the bay') and a large residential section above, on the Niagara Escarpment ('up the mountain'). This leads to many of us coming down the escarpment several times a week, and it is my guess that most of us will look out over the bay to gain a feel for the day's air quality. My own air quality index is instantly calculated as I begin to descend into the city, and ranges from high, when I can make out the high-rise buildings of Burlington or even Toronto's CN Tower ('perfect'), to medium, when the Burlington Skyway Bridge is visible ('hazy'), to low, when even the McMaster Health Sciences Centre cannot be clearly seen ('ugh'). Because we are subject to temperature inversions, one day in three, there is a large variation in visibility that makes this exercise well worthwhile, especially because more scientific (but probably no more valid) measurements of air quality are less available or less publicized nowadays. On one particularly smoggy day recently, an item dealing with the Ontario Medical Association's (OMA's) report card on air quality (1) was on the radio at the same time that my calculation reached an all-time low, prompting me to seek out the text of the report on the OMA's Web site (www.oma.org). It makes interesting reading, especially if one follows it by reading the Ontario Ministry of the Environment's Web site (www.airqualityontario.com).

For someone who lives in Hamilton, the OMA's report held a number of surprises, the main one being that the ambient ozone levels in Hamilton are among the lowest in the province. In general, ozone levels have shown a small but gradual increase, averaging $0.5 \%$ more per year between 1980 and 1998. The differences in ozone levels between communities are very interesting; for example, the small town of Tiverton, Ontario, on the shore of Lake Huron, has an ozone level that is twice that of Hamilton's. Ozone is produced through photochemical reactions involving nitrogen oxides and hydrocarbons in sunlight; thus, it appears as though the ingredients are produced in the urban environment, and the products gradually accumulate and drift into rural areas - an ironic and sobering thought for urban parents who send their children to camp partly to experience the joys of living in the fresh air of the countryside. A bias that I will admit to is the belief that these increases are related to increases in heavy duty traffic on Ontario's

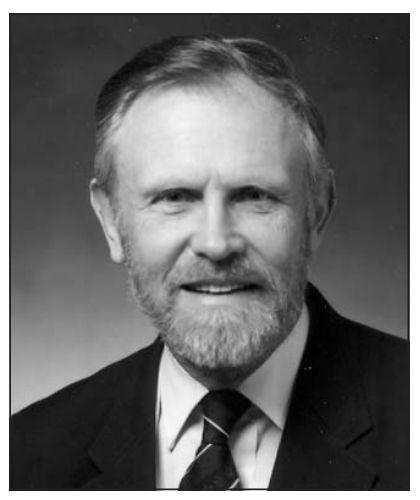
roads, but I could find no data on this.

The OMA's report highlights the reductions in nitrogen oxides and sulphur oxides between 1989 and 1994 without change thereafter, similar trends in ambient concentrations of small particulates, and a gradual increase in temperature between 1945 and the present of about $0.8^{\circ} \mathrm{C}$. The report reviews the recommendations made in 1998, when the first OMA's report on air quality was published (2), and constructs a report card that shows that significant measures have been taken in response to only about one-third of its recommendations.

Again related to my biases, the ministry's Web site indicates that heavy duty vehicles that are over three years old have to undergo emission testing; however, it may be significant that this part of the site has not been updated since September 1999, when a 'temporary' shortage of testing facilities was noted. The OMA's recommendations appear only once on this Web site, where a proposed cap on industrial emissions is called 'unrealistic', without any indication as to why.

Most of the health effects of poor air quality were well known as long as 30 years ago (3). The progress documented in the OMA's report is, thus, very discouraging; the passing of Clean Air Acts in Europe and North America has not been followed by the reduction in pollution that their proponents probably predicted. Also discouraging is the lack of critical 
media attention associated with episodes of severe pollution, such as the one we have just lived through; perhaps this, too, is in part related to the cities being largely responsible for the emissions, and the smaller, less economically and politically powerful rural communities appearing to bear the brunt of the effects. While the 'smog alerts' are publicized, they are based on thresholds of the air quality index - a 'smog watch' is issued when there is a $50 \%$ chance that the air quality index will exceed 50 ('poor') in the next three days, and a 'smog advisory' is issued when this criteria applies to the next $24 \mathrm{~h}$. We know that vulnerable individuals are affected by levels considerably lower than the 'poor' threshold. Better public reporting and education, and greater political pressure are needed if progress is to be made in the continuing war on the health effects of air pollution. Opposition to control measures, based on the supposed negative economic effects, is well funded and politically strong. David Bates has emphasized the role of epidemiology, and analyzed the often conflicting roles of industry, government and the public in achieving success (4).

The OMA's report ends with four recommendations - first, there is a need to reduce the concentration of ozone and particulates in Ontario's air; second, the approach should be based on health effects rather than thresholds; third, the focus should be on ambient concentrations of ozone and particulates rather than on emission data; and finally, public access to real time air pollution data should be improved.

The summary of the Royal College of Physician's report (3) reminds us that a law was passed to limit air pollution as long ago as 1273; legal and political efforts to stem its health effects continue to have only a limited effect. Personally, I am glad that some portion of my OMA membership fees are being used to keep the issues in the public eye.

\section{REFERENCES}

1. Ontario Medical Association. Ontario's Air: Years of Stagnation. $<$ www.oma.org > Version current at July 19, 2001.

2. Boadway BT, MacPhail J, Jacobson C. Ontario Medical Association position paper on health effects of ground-level ozone, acid aerosols and particulate matter. Can Respir J 1998;5:367-84.

3. Royal College of Physicians of London. Air Pollution and Health. London: Pitman Medical, 1970.

4. Bates DV. Environmental Health Risks and Public Policy: Decision Making in Free Societies. Vancouver: UBC Press, 1994. 


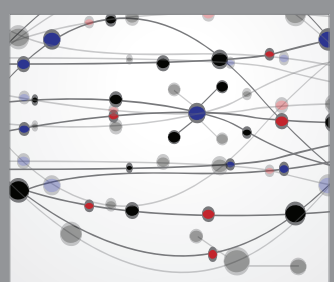

The Scientific World Journal
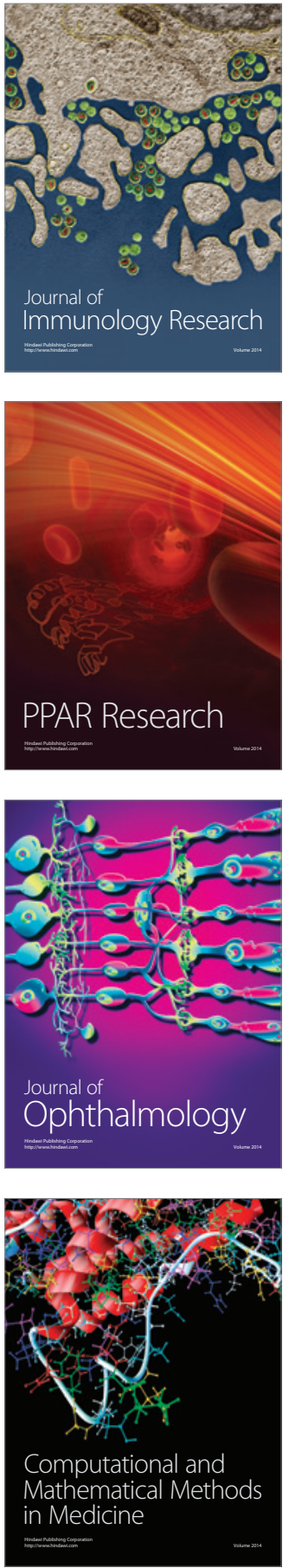

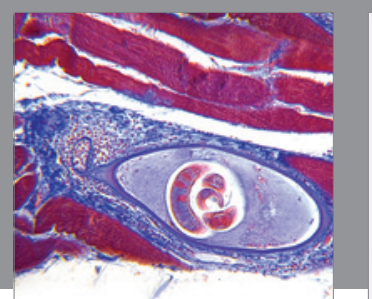

Gastroenterology Research and Practice

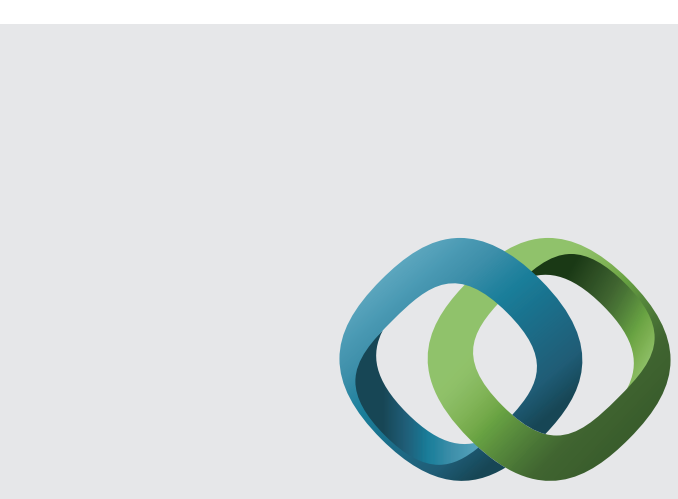

\section{Hindawi}

Submit your manuscripts at

http://www.hindawi.com
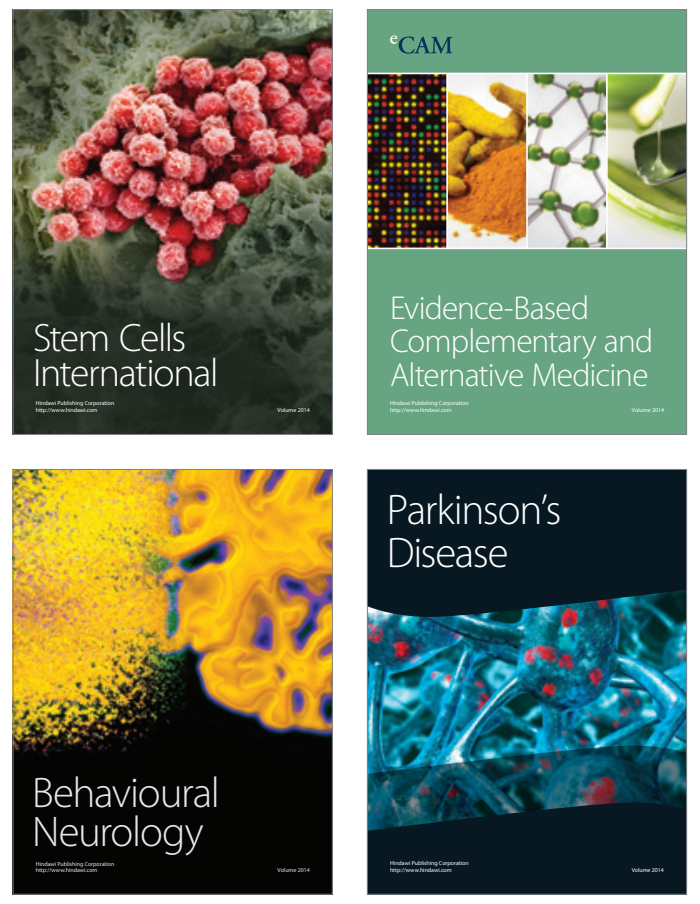
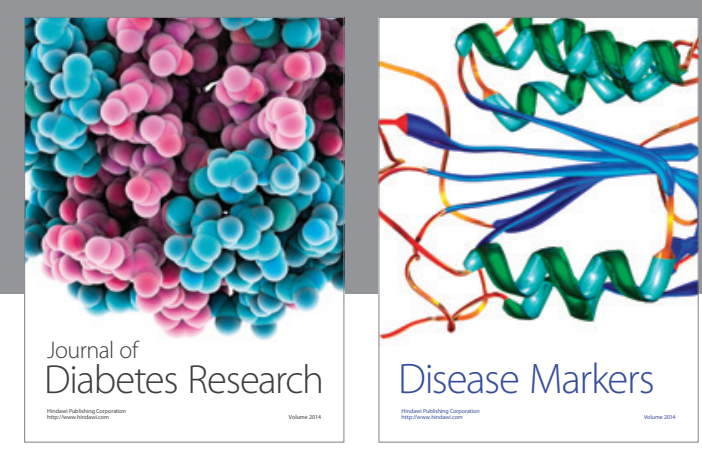

Disease Markers
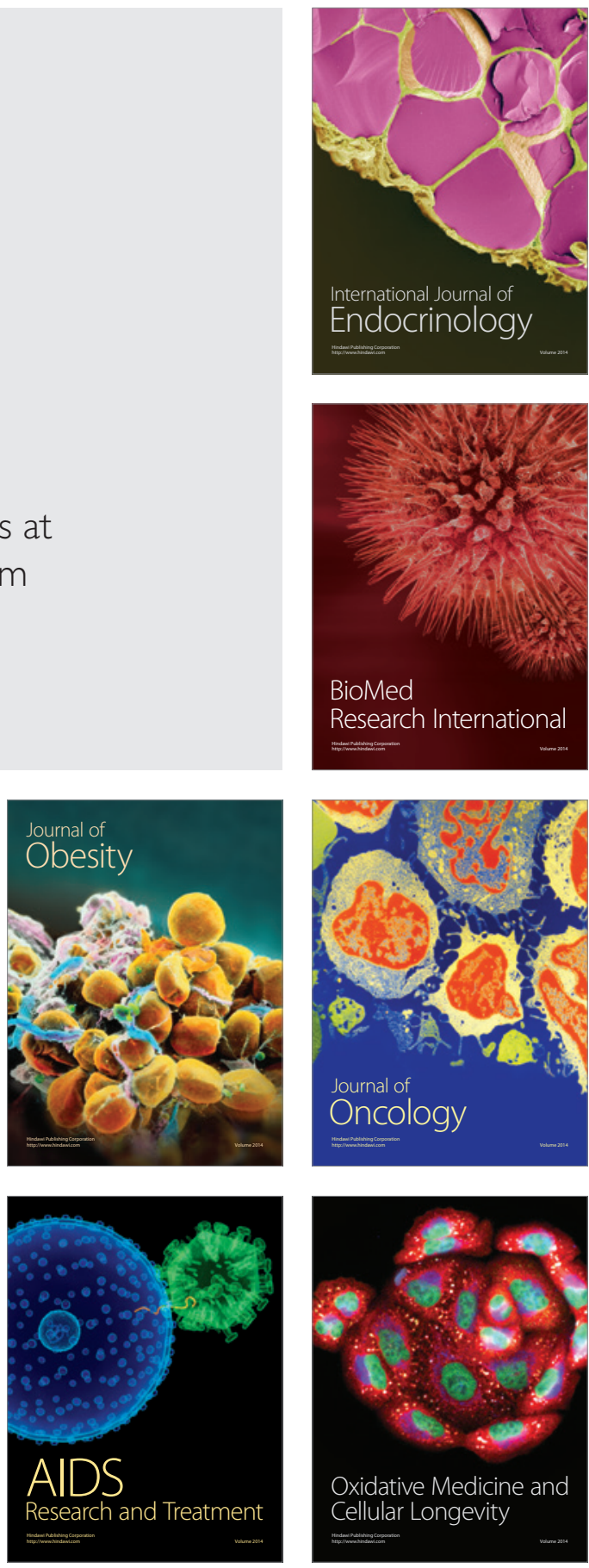